\section{Segurança alimentar em famílias indígenas Teréna, Mato Grosso do Sul, Brasil}

\author{
Food security in Teréna indigenous families, \\ Mato Grosso do Sul, Brazil
}

\footnotetext{
${ }^{1}$ Centro de Ciências Biológicas e da Saúde, Universidade Federal do Mato Grosso do Sul, Campo Grande, Brasil.

2 Centro de Ciências Exatas e Tecnológicas, Universidade Federal do Mato Grosso do Sul, Campo Grande, Brasil. 3 Faculdade de Ciências Médicas, Universidade Estadual de Campinas, Campo Grande, Brasil.

Correspondência T. Fávaro Departamento de Saúde Coletiva, Centro de Ciências Biológicas e da Saúde, Universidade Federal do Mato Grosso do Sul. Cidade Universitária, C. P. 549, Campo Grande, MS 79070-900, Brasil. thatifavaro@uol.com.br
}

\begin{abstract}
This study aims to describe the food security situation among Teréna families in the villages of Água Azul, Olho D'Água, and Oliveiras in Mato Grosso do Sul State, Brazil. The Brazilian Food Insecurity Scale was adapted to 15 questions that reflect food insecurity at different levels of intensity. A survey was conducted in the villages with 49 families that had under-five children. Information was obtained on income, family size, maternal education, and children's food intake. $75.5 \%$ of families showed some level of food insecurity (22.4\% low, 32.7\% moderate, and $20.4 \%$ high). A large percentage (67.3\%) of the families live with fear of lack of food. Onefourth of women had experienced hunger during the month prior to the survey, and 14.3\% (7) reported the same condition for children in the household. More serious food insecurity was observed in families with lower per capita income and lower maternal education, more family members, and more children per family group in which the children's diet was insufficient, especially in protein and iron.
\end{abstract}

Food Security; Food Consumption; South American Indians; Nutrition Surveys

\author{
Thatiana Fávaro 1 \\ Dulce Lopes Barboza Ribas 1 \\ José Roberto Zorzatto 2 \\ Ana Maria Segall-Corrêa 3 \\ Giseli Panigassi 3
}

\section{Introdução}

A definição de segurança alimentar adotada pelo governo brasileiro, pelo Conselho Nacional de Segurança Alimentar e Nutricional (CONSEA) e que serve como base para a definição de políticas públicas de promoção da segurança alimentar é descrita como o acesso regular e permanente a alimentos de qualidade, em quantidade suficiente, sem comprometer o acesso a outras necessidades essenciais, tendo como princípio práticas alimentares promotoras de saúde, que respeitem a diversidade cultural e que sejam sociais, econômicas e ambientalmente sustentáveis 1 . O direito humano à alimentação adequada está previsto na Declaração Universal dos Direitos Humanos ${ }^{2}$. Disponibilidade monetária, estado nutricional e situação de acesso aos alimentos são freqüentemente utilizados como indicadores de insegurança alimentar de uma população, entretanto estar acima da linha de pobreza, com bom estado nutricional e livre de fome não é garantia suficiente de alcance da segurança alimentar e nutricional 3,4.

Pesquisadores de várias instituições brasileiras validaram um método para a avaliação da insegurança alimentar em população brasileira não institucionalizada residente em área urbana (Escala Brasileira de Insegurança Alimentar - EBIA) 5, embasados em um instrumento utilizado pelo United States Department of Agriculture (USDA) 6, que, por sua vez, tem como principal referência a escala proposta por Radimer et 
al. 7. O instrumento, composto por 15 perguntas, permite a avaliação da segurança alimentar intrafamiliar por meio da percepção do entrevistado. As questões incluem a preocupação e a ansiedade da família em relação à obtenção dos alimentos, perpassando pelo comprometimento da qualidade e quantidade da dieta de adultos e crianças, alcançando situações mais graves, com restrições quantitativas de alimentos para todos os membros da família 5,8.

Por intermédio da escala, é possível verificar que a insegurança alimentar atinge de maneira diferenciada os diversos integrantes, adultos e crianças, de uma mesma família, em que as crianças seriam privilegiadas na oferta dos alimentos em detrimento dos adultos, refletindo a gravidade da situação de insegurança alimentar grave quando o alimento está escasso entre os mais jovens da família 9,10. Esse modo de avaliar insegurança alimentar motivou inúmeros estudos de validação do instrumento para diferentes realidades, e os autores são unânimes em afirmar a necessidade de adaptar o método para subgrupos populacionais 11,12,13,14.

No Brasil, os povos indígenas estão expostos a transformações ambientais e sócio-econômicas, que os colocam em situação de alta vulnerabilidade frente a problemas de ordem alimentar e nutricional. Nesse sentido, estudos pontuais realizados em comunidades indígenas revelam a fragilidade de muitos povos frente às conseqüências das carências alimentares, como a elevada prevalência de nanismo nutricional em crianças menores de cinco anos, também favorecida por precárias condições de saneamento, entre outros determinantes 15,16,17. Pesquisas realizadas com indígenas da etnia Teréna, no Mato Grosso do Sul, apontam a gravidade da situação nutricional desse povo, refletida na elevada prevalência de nanismo nas crianças, monotonia alimentar e abandono das práticas tradicionais de cultivo, que no passado contribuíam para a diversidade dos alimentos consumidos e relativa garantia da segurança alimen$\operatorname{tar} 18,19$.

Considerando a importância da realização de estudos que avaliem a situação de segurança alimentar nos povos indígenas e a necessidade de que eles forneçam informações úteis para construir indicadores de condições de vida e saúde, este estudo teve como objetivo determinar a prevalência da insegurança alimentar em famílias Teréna com crianças menores de sessenta meses na composição, assim como descrever o consumo alimentar das crianças e a situação sócio-econômica destas famílias.

\section{Métodos}

Foi realizado um estudo transversal descritivo, no período de abril a dezembro de 2004, na Área Indígena Buriti, pertencente aos municípios de Dois Irmãos do Buriti e Sidrolândia, Mato Grosso do Sul, Brasil, e que possui oito aldeias distribuídas em uma área de 2.090 hectares. O estudo envolveu famílias residentes nas aldeias Olho D'Água, Água Azul e Oliveiras, sendo estas aldeias selecionadas por demanda espontânea, levando-se em consideração aspectos de acesso e interesse das famílias com crianças em participar do estudo.

Foram identificadas 49 famílias com crianças menores de sessenta meses. Todas foram visitadas e aceitaram participar do estudo. Mães ou responsáveis responderam um módulo de caracterização sócio-econômica da família, um módulo sobre a percepção da segurança/insegurança alimentar (escala adaptada) e um módulo sobre o consumo alimentar das crianças.

Para a identificação da renda mensal per capita familiar, foram investigados todos os rendimentos da família no mês anterior à entrevista, em Reais. Quando a família comercializava parte ou toda a produção agrícola, os recursos provenientes da venda dos produtos foram considerados mensalmente. $\mathrm{O}$ valor recebido foi rateado por 12 meses, para fins de cálculo de renda mensal per capita familiar média. $\mathrm{O}$ valor estimado da cesta básica de alimentos foi agregado à renda, assim como os valores recebidos por meio de programas governamentais de distribuição direta de renda. O valor da renda mensal per capita familiar obtido foi transformado em faixas de salário mínimo.

A escolaridade das entrevistadas foi observada em anos de estudo completos e agrupados em "0 a 3 anos" e "mais de 3 anos", considerado o último ano letivo completo. As mulheres foram questionadas quanto à idade, número de filhos e número de membros da família residentes no domicílio.

O instrumento de coleta de dados utilizado para a avaliação da segurança alimentar foi derivado da EBIA 5. Inicialmente, o instrumento foi revisado pelo Grupo de Pesquisa em Saúde e Nutrição de Populações da Universidade Federal do Mato Grosso do Sul, com experiência de mais de dez anos com a população estudada, e posteriormente foi apresentado e discutido em um grupo focal realizado em aldeia da Área Indígena Buriti. O grupo focal foi composto por oito participantes selecionados com a colaboração do agente indígena de saúde (AIS) pelos critérios de: terem um ou mais filhos com idade menor que sessenta meses e desejarem participar do estudo. Foram explorados os termos contidos 
na escala previamente adaptada pelo grupo de pesquisa. Após a exposição e discussão de todos os termos, as perguntas foram reorganizadas com as sugestões resultantes do grupo, e a escala apresentada novamente aos participantes, a fim de que fosse avaliado o entendimento de cada questão e suas possibilidades de resposta.

O escala adaptada foi composta de 15 perguntas fechadas, com respostas positivas e negativas, relativas à percepção das entrevistadas sobre a situação alimentar vivida pela família ao longo do mês anterior à entrevista. As respostas foram codificadas conforme o proposto por Segall-Corrêa et al. 5 , considerando o processo de insegurança alimentar crescente, passando pela preocupação com a falta de alimentos, pelo comprometimento da qualidade da dieta e por fim pela redução quantitativa da alimentação. Para as respostas positivas, foi atribuído o valor "1", e para as respostas negativas o valor "0", resultando em um escore com amplitude de 0 a 15 pontos. A soma dos escores resultantes foi classificada em quatro níveis: "0" segurança alimentar; "1-5" insegurança alimentar leve; "6-10" insegurança alimentar moderada e "11-15" insegurança alimentar grave.

O consumo alimentar das crianças foi avaliado por meio do método recordatório 24 horas, referente ao dia anterior à pesquisa, registrando todos os alimentos consumidos e quantidades desde a primeira refeição da manhã até a última refeição da noite, identificado em nível individual por meio de informações declaradas pela mãe ou responsável. Foi sorteada uma criança por família, por meio de sorteio simples. Foram mensuradas as quantidades consumidas de calorias, proteínas, vitaminas A e C, cálcio e ferro, por intermédio do programa Diet Pro (Agromídia, Viçosa, Brasil) e comparada com as recomendações, sendo que o consumo alimentar foi considerado adequado quando atingiu $90 \%$ ou mais das recomendações 20,21,22,23,24. Os alimentos consumidos foram agrupados em frutas, verduras, leite, ovos, leguminosas, carnes e cereais. Tanto a proporção de adequação como o consumo diário dos grupos de alimentos foram posteriormente comparados com a condição de segurança e insegurança alimentar da família.

Para verificar a existência de associação entre segurança/insegurança alimentar segundo as variáveis sócio-econômicas e de consumo, foi utilizado o teste qui-quadrado ao nível de significância $\alpha=5 \%$. Na impossibilidade do uso do teste qui-quadrado, usou-se o teste Exato de Fisher. O cálculo da odds radio (OR) e respectivo intervalo de confiança (IC95\%) foi utilizado para mostrar a magnitude da associação entre segurança/ insegurança alimentar e as condições sócio-eco- nômicas. A confiabilidade da EBIA adaptada foi feita por meio do coeficiente $\alpha$-Cronbach. Os programas utilizados foram SPSS for Windows versão 11.5 (SPSS Inc., Chicago, Estados Unidos) e BioEstat versão 3.0 (Sociedade Civil Mamirauá, Manaus, Brasil).

Os trabalhos iniciaram-se após autorização das lideranças das aldeias, recebimento do parecer favorável do Comitê de Ética em Pesquisa da Universidade Federal do Mato Grosso do Sul, da Comissão Nacional de Ética em Pesquisa (CONEP) e consentimento livre e esclarecido dos participantes.

\section{Resultados}

\section{Condições sócio-econômicas das famílias}

Em média a renda mensal per capita da família foi de $\mathrm{R} \$ 62,44$ (sessenta e dois Reais e quarenta e quatro centavos) com desvio padrão de $\mathrm{R} \$ 83,53$ (oitenta e três Reais e cinqüenta e três centavos), evidenciando uma alta variabilidade na renda. A distribuição da renda é mais bem visualizada quanto descrita pelos quartis $(\mathrm{Q} 1=\mathrm{R} \$ 25,80$; $\mathrm{Q} 2$ $=\mathrm{R} \$ 33,70$ e $\mathrm{Q} 3=\mathrm{R} \$ 69,50$ ), com pouco mais de $25 \%$ das famílias vivendo com renda mensal per capita maior de um quarto de salário mínimo. Todas as famílias entrevistadas referiram receber a cesta básica de alimentos e 71,4\% (35) das famílias estavam inseridas em programas de distribuição direta de renda, como Vale-Gás, Bolsa-Escola, Bolsa-Alimentação ou Bolsa-Família, sendo estes integrantes do pool de benefícios do Programa Fome Zero, do Governo Federal à época do estudo. Foram identificadas três formas de obtenção de alimentos pelas famílias: recebimento de cesta básica pelo Programa de Segurança Alimentar Estadual, plantio de lavouras e aquisição de produtos na cidade mais próxima ou em mercearias nas próprias.

Nas 49 famílias que fizeram parte do estudo, $59,2 \%$ (29) das mulheres entrevistadas possuíam até três anos de escolaridade; 16,3\% (8) informaram nunca terem freqüentado a escola. O tempo de escolaridade foi em média de 3,8 anos, com desvio padrão de 3,4 anos. Mães ou responsáveis com idades entre 20 e 29 anos representaram $51 \%$ (25) das entrevistadas, com média de idade de 30,2 anos e desvio padrão de 10,0 anos, variando de 18 a 74 anos. Além das mães biológicas das crianças, foram identificadas duas avós, com 45 e 74 anos. Aproximadamente a metade das mulheres (24) afirmou ter entre 1 a 3 filhos, sete mulheres $(14,3 \%)$ referiram terem mais de sete filhos. A média do número de filhos verificado foi de 3,9 filhos e desvio padrão de 2,6 filhos. 


\section{Insegurança alimentar}

A Tabela 1 apresenta a EBIA adaptada à população estudada, com a freqüência de respostas positivas a cada item. O valor do teste $\alpha$-Cronbach $(0,93)$ indicou confiabilidade satisfatória do instrumento.

Nas famílias estudadas, a prevalência segurança alimentar foi de $24,5 \%$, de insegurança alimentar leve foi de $22,4 \%$, moderada $32,7 \%$ e grave em $20,4 \%$. Famílias com segurança alimentar foram aquelas que não apresentaram dificuldades em manter o consumo alimentar em quantidade e qualidade suficientes; insegurança alimentar leve correspondeu a famílias preocupadas com a falta de alimentos e com comprometimento quanto à qualidade da dieta; insegurança alimentar moderada esteve presente quando, além de problemas com a qualidade da dieta, existe também a restrição quantitativa no consumo de adultos e insegurança alimentar grave representou a forma mais severa, em que crianças passam por restrições alimentares, em qualidade e quantidade.

Nas famílias em que a escolaridade materna é menor (<3 anos), a proporção de insegurança alimentar leve e moderada é significativamente maior $(73,1 \%)$ que entre as famílias com a escolaridade materna maior $(43,5 \%)(p=0,015)$. Também foram observadas diferenças significativas nas demais variáveis sócio-econômicas segundo a situação de segurança/insegurança alimentar. Na Tabela 2, observa-se que, para todas as variáveis sócio-econômicas utilizadas neste estudo, a situação de insegurança alimentar moderada e grave está presente em maior proporção e com maior possibilidade de ocorrência nas famílias com menor renda, menor escolaridade materna e maior densidade.

A preocupação com a falta do alimento foi relatada como realidade para $67,3 \%$ (33) das mulheres entrevistadas e $57,1 \%$ (28) afirmaram que os gêneros alimentícios realmente acabaram antes que fosse possível a obtenção de novo estoque, no mês anterior à entrevista. Os itens da escala que se referiam a restrições na qualidade da dieta oferecida à família foram aqueles que apresentaram maior proporção de respostas positivas, em torno de $60 \%$, ou seja, essas mulheres afirmaram que não foi possível o preparo de uma "comida boa" para a sua família, por falta de alimentos considerados adequados para tanto. Em proporções semelhantes tanto para a entrevistada como para as crianças (51\% e 49,9\%) foi relatado o consumo de menor quantidade de alimentos daquela considerada como suficiente. Quanto a deixar de realizar uma das três refeições principais durante o dia, as crianças foram parcialmente protegidas, visto que aproximadamente metade das entrevistadas (23) afirmou que esta situação ocorreu com adultos e nove afirmaram que crianças foram privadas de, ao menos, uma refeição. Um quarto das mulheres entrevistadas afirmou ter passado por situações de fome, no mês anterior à entrevista e 14,3\% (7) referiram que a mesma situação aconteceu com as crianças da casa (Tabela 1).

Embora 49 crianças tenham sido sorteadas para a análise do consumo, apenas 36 crianças foram incluídas, já que em quatro casos a entrevistada não se sentiu capaz de fornecer informações fidedignas sobre a alimentação da criança, e nove crianças possuíam como alimentação predominante o aleitamento materno, o que inviabilizou a mensuração e análise do consumo.

O consumo de cereais, representado basicamente pelo arroz, e de leguminosas, representadas pelo feijão, foi observado na maioria das dietas analisadas e faziam parte das três refeições diárias realizadas pelas crianças. As diferenças na proporção de crianças que consumiram frutas foram significativas quando comparados os grupos com segurança alimentar/insegurança alimentar leve e insegurança alimentar moderada/grave (55,5\% vs. 16,6\%; $\mathrm{p}=0,015)$, assim como para o consumo de carnes $(72,2 \%$; vs. $38,8 \% ; \mathrm{p}=$ 0,044). Observou-se, pela OR e IC95\% superior a 1, maior chance do consumo destes alimentos entre crianças de famílias com segurança alimentar e insegurança alimentar leve. Verduras foram consumidas com pouca freqüência por ambos os grupos [16,6\% (segurança alimentar/ insegurança alimentar leve) vs. 11,1\% (insegurança alimentar moderada/grave); $\mathrm{p}=0,6688$ ]. O consumo de leite foi superior por crianças de famílias classificadas com insegurança alimentar moderada/grave quando comparadas a famílias com segurança alimentar/insegurança alimentar leve $(33,3 \%$ vs. $16,6 \%$; $\mathrm{p}=0,2821)$, embora sem diferença significativa. A presença de leite dentre essas famílias pode ser justificada em função da distribuição de leite em pó, realizada pela Fundação Nacional de Saúde (FUNASA) para crianças com baixo peso, que possivelmente se concentra nas famílias com insegurança alimentar moderada/grave.

O consumo não adequado em relação às calorias foi verificado em $66,6 \%$ das crianças pertencentes às famílias com segurança alimentar/insegurança alimentar leve e em $94,4 \%$ das crianças em famílias com insegurança alimentar moderada/grave, porém sem diferença estatisticamente significativa $(\mathrm{p}=0,087)$. Foi observada menor proporção de crianças com consumo protéico inadequado em famílias classificadas em segurança alimentar/insegurança alimen- 
Tabela 1

Freqüência de respostas positivas aos itens da Escala Brasileira de Insegurança Alimentar adaptada para a população indígena

Teréna. Área Indígena Buriti, Mato Grosso do Sul, Brasil, 2004.

\begin{tabular}{|c|c|c|}
\hline Item & Durante o último mês & $\%$ \\
\hline 1 & $\begin{array}{l}\text { Aconteceu alguma vez de a senhora ficar preocupada que a comida de sua casa fosse acabar antes que chegasse a } \\
\text { outra cesta básica ou que alguém da casa tivesse dinheiro para comprar comida ou que tivesse produção da roça? }\end{array}$ & 67,3 \\
\hline 2 & Aconteceu de a comida de sua casa acabar antes do final do mês? & 57,1 \\
\hline 3 & Aconteceu de a senhora ficar sem recursos para ter uma comida boa em casa? & 63,3 \\
\hline 4 & Aconteceu de a senhora ter que se arranjar com apenas alguns alimentos para comer porque não tinha recursos? & 63,3 \\
\hline 5 & $\begin{array}{l}\text { Aconteceu de a senhora não poder dar às crianças uma comida boa porque a cesta havia acabado e não havia } \\
\text { produção na roça ou dinheiro para comprar? }\end{array}$ & 57,1 \\
\hline 6 & Aconteceu de as crianças comerem menos comida porque não tinha comida suficiente em casa? & 49,0 \\
\hline 7 & $\begin{array}{l}\text { Aconteceu de algum adulto da casa ou a senhora deixar de comer (de manhã, no almoço ou no jantar) porque } \\
\text { não tinha comida suficiente em casa? }\end{array}$ & 46,9 \\
\hline 8 & Aconteceu de a senhora comer menos do que devia por que tinha pouca comida em casa? & 51,0 \\
\hline 9 & Aconteceu de a senhora sentir fome e não ter nada para comer em casa? & 24,5 \\
\hline 10 & A senhora perdeu peso (emagreceu) porque ficou sem ter o que comer em casa? & 16,3 \\
\hline 11 & $\begin{array}{l}\text { Aconteceu de a senhora ou algum adulto da cada ficar o dia inteiro sem comer ou comer só uma vez no dia } \\
\text { porque não tinha comida em casa? }\end{array}$ & 16,3 \\
\hline 12 & Aconteceu de a senhora ter que servir menos comida para as crianças porque tinha pouca comida? & 44,9 \\
\hline 13 & Aconteceu de as crianças deixarem de comer (de manhã, no almoço ou no jantar) porque não tinha comida suficiente em casa? & 18,4 \\
\hline 14 & Aconteceu de as crianças ficarem com fome e não comeram porque não tinha comida em casa? & 14,3 \\
\hline 15 & Aconteceu de as crianças ficarem o dia inteiro sem comer porque não tinha comida em casa? & 10,2 \\
\hline
\end{tabular}

Tabela 2

Prevalência de segurança/insegurança alimentar segundo variáveis sócio-econômicas de famílias Teréna. Área Indígena Buriti,

Mato Grosso do Sul, Brasil, $2004(n=49)$

\begin{tabular}{|c|c|c|c|c|c|c|}
\hline \multirow[t]{2}{*}{ Variáveis } & \multicolumn{2}{|c|}{$\begin{array}{l}\text { Insegurança alimentar } \\
\text { moderada e grave }\end{array}$} & \multicolumn{2}{|c|}{$\begin{array}{c}\text { Segurança alimentar e } \\
\text { insegurança alimentar leve }\end{array}$} & \multirow[t]{2}{*}{ OR bruto } & \multirow[t]{2}{*}{ IC95\% } \\
\hline & n & $\%$ & $n$ & $\%$ & & \\
\hline \multicolumn{7}{|c|}{ Número de membros na família } \\
\hline$>5$ & 19 & 73,1 & 10 & 43,5 & & \\
\hline $0-5$ & 7 & 30,4 & 13 & 56,5 & 3,53 & $1,07-11,67$ \\
\hline \multicolumn{7}{|c|}{ Escolaridade materna (anos) * } \\
\hline $0-3$ & 19 & 73,1 & 10 & 43,5 & & \\
\hline$>3$ & 7 & 30,4 & 13 & 56,5 & 3,53 & $1,07-11,67$ \\
\hline \multicolumn{7}{|c|}{ Número de filhos } \\
\hline$>3$ & 18 & 69,2 & 7 & 30,4 & & \\
\hline $0-3$ & 8 & 30,8 & 16 & 69,6 & 5,14 & $1,52-17,38$ \\
\hline \multicolumn{7}{|c|}{ Renda familiar per capita (salário mínimo) } \\
\hline $0-0,25$ & 23 & 88,5 & 12 & 52,2 & & \\
\hline$>0,25$ & 3 & 11,5 & 11 & 47,8 & 7,03 & $1,64-30,11$ \\
\hline
\end{tabular}

* Incluindo mães e avós. 
tar leve comparadas às famílias com insegurança alimentar moderada/grave $(5,6 \%$ vs. $44,4 \%$; $\mathrm{p}=0,0178$ ). Já em relação ao consumo de micronutrientes, apenas para o ferro observou-se diferença significativa $(p=0,001)$ em relação aos níveis de in/segurança alimentar, estando adequado na dieta alimentar de $61,1 \%$ e $11,1 \%$, respectivamente, para crianças de famílias com segurança alimentar/insegurança alimentar leve e com insegurança alimentar moderada/grave. O consumo de vitamina A foi considerado inadequado em $88,8 \%$ das dietas independentemente do nível de insegurança alimentar, tal como o consumo inadequado de vitamina C em 38,9\% das dietas analisadas. Em consonância com o consumo superior de leite por crianças de famílias com maior comprometimento quando comparadas à insegurança alimentar, o consumo de cálcio foi superior em famílias com insegurança alimentar moderada/grave $(12,5 \%$ vs. $27,7 \%$; $\mathrm{p}=$ 0,4018 ), e de maneira geral o consumo foi considerado adequado apenas para $19,4 \%$ (7) das crianças.

\section{Discussão}

Famílias classificadas na categoria segurança alimentar corresponderam à cerca de um quarto do universo estudado, e mesmo não havendo estudos que avaliem a segurança alimentar em povos indígenas da forma como foi vista no presente, é alarmante a realidade encontrada, na qual grande parte das famílias está exposta às privações tanto na qualidade como na quantidade da dieta. Somando-se a isso, o comprometimento social dessas famílias, como a baixa renda, condições precárias de moradia e extensão territorial limitada para o plantio 19 , revelam um quadro de instabilidade, com grande parte das famílias convivendo com o medo da falta do alimento.

Melgar-Quiñonez et al. 25, ao estudarem a percepção e a experiência de insegurança alimentar em latino-americanos vivendo na California, Estados Unidos, explicaram a insegurança alimentar como um processo administrado dentro da família, com estratégias para o enfrentamento da situação de dificuldade de acesso aos alimentos, que incluía a prioridade de consumo para as crianças e a preferência por alimentos mais baratos. Os autores concordaram que a situação de insegurança alimentar é sentida em diferentes níveis de severidade pela família. Comportamento similar pode ser percebido na população aqui estudada, quando avaliada a proporção de respostas positivas às questões da escala. Perguntas de maior severidade foram organizadas de forma decrescente na escala e a proporção de respostas afirmativas, referentes ao comprometimento quantitativo da dieta das crianças, diminui gradativamente.

Estudo realizado em Campinas com 195 famílias contendo idosos, e utilizando a EBIA, encontrou a prevalência de $52 \%$ das famílias com algum grau de insegurança alimentar. Nessas famílias, os idosos apresentaram renda e escolaridade menores que nas famílias com segurança alimentar 26. Ainda que sejam ciclos de vida opostos, baixa escolaridade e renda estiveram associadas com a insegurança alimentar em famílias Teréna com crianças menores de sessenta meses. A renda per capita de famílias com insegurança alimentar grave foi aproximadamente $70 \%$ menor do que aquelas com segurança alimentar.

Estudo realizado em Hartford, Estados Unidos, que comparou níveis de segurança alimentar em famílias beneficiárias ou não de programas assistenciais e contemplou famílias com crianças menores de sessenta meses, evidenciou que a inserção nos programas de suporte alimentar garantia a segurança alimentar e o consumo de micronutrientes pelas crianças 27 . No presente estudo, onde todas as famílias recebiam a cesta de alimentos e a maioria está inserida em programas de distribuição de renda, não foi observada a mesma relação, e o consumo adequado de micronutrientes também não foi constatado, seja para famílias beneficiárias ou não de programas de distribuição de renda.

O padrão de alimentação observado entre as crianças aponta para sério comprometimento alimentar, em que menos da metade das crianças tem acesso a uma alimentação que ofereça os micronutrientes essenciais para o seu crescimento e desenvolvimento, e nas famílias que foram classificadas como inseguras e com restrições quantitativas de alimentos, o déficit é mais acentuado quanto à ingestão de proteínas e ferro. Fontes protéicas e férricas, especialmente as de origem animal, dependem de disponibilidade financeira para sua aquisição, uma vez que práticas de caça e de pesca são inviáveis na área, sobretudo pela limitação territorial e poluição dos rios. Pode-se supor que, mesmo as frutas, que estão disponíveis em algumas épocas do ano, em pequenas áreas de poucas famílias, e não dependem de disponibilidade financeira para o consumo, não são acessíveis às famílias mais vulneráveis.

Estudos com crianças indígenas chamam a atenção para elevadas prevalências de déficits de massa corporal e estatura 15,16,17, situações desencadeadas particularmente por inadequações no consumo alimentar e pelas precárias condições de vida a que muitos povos estão subme- 
tidos. Em 1999, estudo realizado com crianças Teréna da mesma área indígena do presente estudo, apontou que as mesmas não consumiam alimentos suficientes em fontes de ferro, cálcio e retinol, e $16 \%$ estavam com retardo de crescimento 19 .

O plantio de lavouras foi identificado como uma forma importante de obtenção de alimentos na área estudada, porém não fez parte do objetivo deste estudo uma análise profunda sobre as formas de produção e área destinada para plantio. No entanto, percebe-se a valorização da agricultura e o predomínio do plantio de mandioca, arroz, feijão e milho. Segundo informações obtidas com os chefes das famílias, a produção é pequena devido à limitação territorial e os produtos são preferencialmente utilizados para a subsistência familiar. Quando destinado à venda, nem sempre alcança preços compatíveis com o mercado, por conta da dificuldade de transporte da mercadoria até a cidade, fazendo com que o comprador se desloque até a área e imponha preços mais baixos.

\section{Considerações finais}

De maneira geral, as famílias que apresentaram situações mais graves de insegurança alimentar foram aquelas com menor renda mensal per capita, menor escolaridade materna, maior densidade familiar e cuja dieta das crianças era insuficiente, sobretudo em proteínas e ferro. Por outro lado, o consumo alimentar mostrou-se comprometido para grande parte das crianças, independentemente do nível de segurança/insegurança alimentar.

Avaliou-se uma das faces da insegurança alimentar dentro de uma complexa rede causal, em que a segurança alimentar foi entendida como a garantia à presença de alimentos em quantidade e qualidade para todos os membros da família, segundo a percepção da entrevistada. $\mathrm{O}$ acesso aos alimentos por uma parcela dessa população não representa, no entanto, que tais famílias e suas crianças estejam protegidas. Assim, partindo-se do conceito de segurança alimentar, apresentado no início deste artigo, é fato que ela não é garantida para essa população, uma vez que, mesmo para aquelas famílias que se autoperceberam seguras, existe ainda a possibilidade de elas estarem sendo mantidas em razão do recebimento da cesta básica de alimentos, fato perpetuador da dependência por ações emergenciais, as quais se transformam em permanentes quando mantidas por longo período. Por mais que seja considerada como uma medida eficaz de combate à fome, observa-se que os alimentos distribuídos não correspondem aos hábitos alimentares dessa população, e a quantidade de alimentos não é compatível com o tamanho médio das famílias.

A utilização da EBIA adaptada mostrou-se uma forma possível de avaliar a situação alimentar das famílias, perpassando desde a preocupação com a falta de alimento no futuro próximo até a redução quantitativa na alimentação. Entretanto a sua utilização com outros grupos deve ser concebida à luz das especificidades de cada povo, e a aplicação da escala tem de ser precedida de etapas prévias de adaptação. A escala construída neste estudo presta-se à avaliação da situação de famílias Teréna moradoras da Área Indígena Buriti.

$\mathrm{O}$ acesso a alimentos de qualidade, em quantidades suficientes e adequadas à cultura alimentar ainda é um obstáculo a ser ultrapassado por essa população. É importante lembrar que o significado da produção de alimentos na cultura Teréna vai além da manutenção do corpo e faz parte do modo de ser Teréna. Nesse sentido, a garantia da terra tantas vezes reivindicada pelas lideranças, assim como outras ações transdisciplinares e a participação comunitária devem ser priorizadas a fim de que possam vir a promover a segurança alimentar e nutricional. 


\section{Resumo}

O presente estudo buscou descrever a situação de segurança alimentar vivenciada por famílias Teréna, das aldeias Água Azul, Olho D'Água e Oliveiras, Mato Grosso do Sul, Brasil. Foi utilizada a Escala Brasileira de Insegurança Alimentar adaptada, com 15 questões que refletem a insegurança alimentar em diferentes níveis de intensidade. Foram investigadas 49 famílias que continham em seu núcleo crianças menores de sessenta meses e obtidas informações sobre renda, densidade familiar, escolaridade materna e consumo alimentar das crianças. A prevalência de famílias com algum grau de insegurança alimentar observada foi 75,5\%, 22,4\% das famílias com insegurança leve, 32,7\% moderada e 20,4\% grave. Grande parte das famílias (67,3\%) convive com o medo de ficar sem alimentos. Um quarto das mulheres entrevistadas afirmou ter passado por situações de fome no mês anterior à entrevista e 14,3\% (7) apontaram que o mesmo ocorreu com as crianças da casa. Situações mais graves de insegurança alimentar foram observadas em famílias com menor renda mensal per capita, menor escolaridade materna, maior densidade domiciliar, maior número de filhos por grupo familiar e cuja dieta das crianças era insuficiente, sobretudo em proteinas e ferro.

Segurança Alimentar e Nutricional; Consumo de Alimentos; Índios Sul-Americanos; Inquéritos Nutricionais

\section{Colaboradores}

T. Fávaro e D. L. B. Ribas participaram da coleta de dados, revisão bibliográfica, análise e discussão do artigo. J. R. Zorzatto, A. M. Segall-Corrêa e G. Panigassi colaboraram na análise e discussão do artigo. Todos os autores contribuíram para a elaboração do artigo final.

\section{Agradecimentos}

Aos moradores das aldeias Água Azul, Olho d'Água e Oliveiras, que receberam a equipe de pesquisa. Aos agentes indígenas de saúde Ester Reginaldo e Sidmar Alcântara, que acompanharam as visitas às residências. Aos alunos José Henrique Cavalheiro, Vânia Paula Stolte, Tatyanne Ferreira da Silva, nutricionista Andreia de Oliveira Massulo e enfermeiras Cacilda Hildebrand e Marcela Aparecida Bertoldi, que participaram da coleta de dados. Ao motorista Adhemar Vilela Moreira que acompanhou todas as viagens às aldeias e auxiliou os trabalhos. Ao técnico-administrativo Hélio Samúdio, que se responsabilizou pelo banco de dados da pesquisa.

Trabalho derivado da Dissertação de Mestrado Segurança Alimentar em Famílias Teréna, Mato Grosso do Sul, Brasil, do Programa de Pós-graduação em Saúde Coletiva da Universidade Federal do Mato Grosso do Sul e baseado em pesquisa de campo financiada pelo Fundo Nacional de Saúde, processo número 2005ND324.

\section{Referências}

1. Conselho Nacional de Segurança Alimentar. Princípios e diretrizes de uma política de segurança alimentar. Brasília: Editora Positiva; 2004.

2. Organização das Nações Unidas. Declaração universal dos direitos humanos. http://www. unhchr.ch/udhr/lang/por.htm (acessado em 13/ Ago/2005).

3. Valente FLS. Fome, desnutrição e cidadania: inclusão social e direitos humanos. Saúde Soc 2003; 12:51-60.

4. Monteiro CA. A dimensão da pobreza, da desnutrição e da fome no Brasil. Estud Av 2003; 48:7-20.

5. Segall-Corrêa AM, Pérez-Escamilla R, Maranha LK, Sampaio MFA, Yuyana L, Alencar F, et al. Projeto: acompanhamento e avaliação da segurança alimentar em famílias brasileiras: validação de metodologia e de instrumento de coleta de informação. Campinas: Departamento de Medicina Preventiva e Social, Universidade Estadual de Campinas/Organização Pan-Americana da Saúde/Ministério da Saúde; 2003. (Relatório Técnico).
6. Bickel G, Nord M, Price C, Hamilton W, Cook J. Measuring food security in the United States: guide to measuring household food security. Alexandria: Office of Analysis, Nutrition, and Evaluation, U.S. Department of Agriculture; 2000.

7. Radimer KL, Olson CM, Greene JC, Campbell CC, Habicht JP. Understanding hunger and developing indicators to assess it in women and children. J Nutr Educ 1992; 24(1 Suppl):36-44.

8. Pérez-Escamilla R, Segall-Corrêa AM, Maranha LK, Sampaio MFA, Marín-León L, Panigassi G. An adapted version of the U. S. Department of Agriculture Food Insecurity Module is a valid tool for assessing household food insecurity in Campinas, Brazil. J Nutr 2004; 134:1923-8.

9. Kendall A, Olson CM, Frongillo Jr. EA. Validation of the Radimer/Cornell measures of hunger and food insecurity. J Nutr 1995; 25:2793-801.

10. Frongillo Jr. EA. Validation of measures of food insecurity and hunger. J Nutr 1999; 129(25 Suppl):506S-9S. 
11. Harrison GG, Storner A, Herman D, Winham DM. Development of Spanish-language version of the U.S. Household Food Security Survey Module. J Nutr 2003; 133:1192-7.

12. Lorenzana PA, Sanjur D. Abbreviated measures of food sufficiency validly estimative the food security level of poor households: measuring household food security. J Nutr 1999; 129:687-92.

13. Albert PL, Sanjur D. The adaptation and validation of the food security scale in a community of Caracas, Venezuela. Arch Latinoam Nutr 2000; 50: 334-40.

14. Alvarado BE, Zunzunegui MV, Delisle H. Validation of food security and social support scales in an Afro-Colombian community: application on a prevalence study of nutritional status in children aged 6 to 18 months. Cad Saúde Pública 2005; 21:724-36.

15. Escobar AL, Santos RV, Coimbra Jr. CEA. Avaliação nutricional de crianças indígenas Pakaanóva (Warí), Rondônia, Brasil. Rev Bras Saúde Matern Infant 2003; 3:457-61.

16. Pícoli RP, Carandina L, Ribas DLB. Saúde maternoinfantil e nutrição de crianças Kaiowá e Guaraní, Área Indígena de Caarapó, Mato Grosso do Sul, Brasil. Cad Saúde Pública 2006; 22:223-7.

17. Coimbra Jr. CEA, Santos RV. Avaliação do estado nutricional num contexto de mudança sócio-econômica: o grupo indígena Suruí do estado de Rondônia, Brasil. Cad Saúde Pública 1991; 7:538-62.

18. Ribas DLB, Philippi ST. Aspectos alimentares e nutricionais de mães e crianças indígenas Teréna, Mato Grosso do Sul. In: Coimbra Jr. CEA, Santos RV, Escobar AL, organizadores. Epidemiologia e saúde dos povos indígenas no Brasil. Rio de Janeiro: Editora Fiocruz/ABRASCO; 2003. p. 73-88.
19. Ribas DLB, Sganzerla A, Zorzatto JR, Philippi ST. Nutrição e saúde infantil em uma comunidade indígena Teréna, Mato Grosso do Sul, Brasil. Cad Saúde Pública 2001; 17:323-44.

20. Institute of Medicine. Dietary reference intakes for vitamin $\mathrm{C}$, vitamin $\mathrm{E}$, selenium and carotenoids. Washington DC: National Academy Press; 2000.

21. Institute of Medicine. Dietary reference intakes for vitamin A, vitamin $\mathrm{K}$, arsenic, boron, chromium, cooper, iodine, iron, manganese, molybdenum, nickel, silicon, vanadium an zinc. Washington DC: National Academy Press; 2001.

22. Institute of Medicine. Dietary reference intakes for energy, carbohydrate, fiber, fat, fatty acids, cholesterol, protein ad amino acids (macronutrients). Washington DC: National Academy Press; 2002.

23. Institute of Medicine. Dietary reference intakes for calcium, phosphorus, magnesium, vitamin D and fluoride. Washington DC: National Academy Press; 1997.

24. National Research Council. Recommended dietary allowances. 10th Ed. Washington DC: National Academy Press; 1989.

25. Melgar-Quinonez H, Kaiser L, Martin A, Metz D, Olivares A. Food insecurity among Latinos in California: a focal groups study. Salud Pública Méx 2003; 45:198-205.

26. Marín-León L, Segal-Corrêa AM, Panigassi G, Maranha LK, Sampaio AFA, Pérez-Escamilla R. A percepção de insegurança alimentar em famílias com idosos de Campinas, São Paulo, Brasil. Cad Saúde Pública 2005; 21:1433-40.

27. Pérez-Escamilla R, Ferris AN, Drake L, Haldeman L, Peranick J, Campbell M, et al. Food stamps are associated with food security and dietary intake of inner-city preschoolers from Hartford, Connecticut. J Nutr 2000; 130:27-117.

Recebido em 02/Fev/2006

Versão final reapresentada em 05/Set/2006

Aprovado em 12/Set/2006 